(Aus dem physiologischen Institut der Universität Wien.)

\title{
Das anatomische Verhalten der Muscularis mucosae in Beziehung zu ihrer physiologischen Bedeutung.
}

Von

stud. med. Bianca Bienenfeld.

In seiner Arbeit "Wie schützt sich der Verdaungstract vor Verletzungen durch spitze Fremdkörper" hat D r. Alf. Ex ner (1) Versuche mitgetheilt, in denen er die interessante Thatsache nachwies, dass die Muscularis mucosae des Darmeanals (wahrscheinlich nebst anderen, auch) die Aufgabe hat, das Eindringen von Fremdkörpern in die Darmschleimhaut zu hindern. Es scheint fürwahr sehr merkwürdig, dass so oft spitze Gegenstände den Verdauungstract passiren, ohne Verletzungen zu verursachen, bietet doch die tägliche Nahrung, das Verschlucken von Knochen und Gräten, den Raubthieren und Raubvögeln wiederholt die Möglichkeit einer Verletzung. In den Mägen körnerfressender Vögel findet man bei Sectionen oft spitze Fremdkörper, ja, Land o is berichtet in seiner Physiologie, dass er Glaskugeln in Splitter zerbrochen fand. Wie A. Exner durch Versuche an lebenden Thieren gezeigt hat, contrahirt sich die durch eine Nadelspitze gereizte Muskulatur der Schleimhaut derart, dass die gereizte Stelle anämisch wird, eine Delle bildet und dem Druck der Spitze ausweicht. Magen und Darm der Thiere pflegen Stecknadeln, die mit dem spitzen Ende voraus eingeführt wurden, umzudrehen, so dass die Nadeln mit dem stumpfen Ende voraus, also ohne schädigende Wirkung, durch den Verdauungstract wandern. Somit besitzt die Darmschleimhaut thatsächlich in ihrer Muskelschicht, die sich vom Oesophagus durch den Magen und den ganzen Darmcanal erstreckt, eine vorzügliche Schutzvorrichtung gegen Verletzungen. Bevor ich auf meine Untersuchungen eingehe, sei es mir gestattet, Einiges über die Entwicklung der Kenntniss dieser Muskelschicht anzuführen. Die erste Mitheilung einer Muskelschicht in der 
Schleimhaut des Duodenums machte 1846 A. Th. Middeldorpf (2) in seiner "Disquisitio de glandulis Brunnianis Vratislaviae". Seine Entdeckung ging aber wieder verloren, und das Verdienst, sie neu aufgefunden zu haben, gebührt Brücke. Es war zwar schon vor ibm dem Franzosen Lacauchie (3) bekannt gewesen, dass die Zotten sich nach dem Tode zusammenziehen. Er hatte aber keine Kenntniss von der Muskulatur, die sich in den Zotten befindet, und schreibt die Contractilität der Darmzotten den Blutgefässen zu. Was Lacauchie am todten Darm bemerkt hatte, sahen Gruby und Delafond (4) an dem des lebenden Thieres. Sie bemerkten eine Bewegung der Darmzotten nach drei Richtungen und sahen die Ursache der Bewegung in den Contractionen des sogen. contractilen Bindegewebes. Erst Brücke (5) veröffentlichte 1851 in den Sitzungsberichten der Wiener Akademie der Wissenschaften die Ergebnisse seiner Untersuchungen, dahin gehend, dass ein bisher völlig unbekanntes Muskelsystem, aus organisehen Muskelfasern bestehend, im Oesophagus, vom Epithel durch eine dünne Bindegewebsschicht getrennt, beginnt und sich durch Dünn- und Dickdarm verfolgen lässt. Fr untersehied im Magen und Darm eine äussere, aus Längsfasern, und eine innere, aus Ringfasern bestehende Schicht. Von dieser ziehen Muskelfasern gegen die Oberfläche der Schleimhaut und bilden in den Zotten ein System von Längsfasern, das bis in das äusserste Fnde der Zotten hineinreicht. Brücke hat auch Messungen der Dicke dieser "inneren Muskelhaut" vorgenommen und sie im Magen 50-100 $\mu$, im Dänndarm höchstens $38 \mu$, im Colon nur $29 \mu$, im Rectum $50 \mu$ und am Anus $200 \mu$ breit gefunden. Unabhängig von Brücke entdeckte Kölliker (6) 1851 bei Untersuchung des Oesophagus, dass sich in dessen Schleimhaut ein grosser Reichthum an glatten Muskelfasern finde und sich dieser Zug von Muskulatur bis in den Magen erstrecke. In der Schleimhaut des Dünn- und Dickdarms sah er keine glatte Muskulatur, ebenso anfangs keine in den Zotten. 1867 aber fand auch er in den Zotten um die Lymphgefässe herum eine dünne Lage von längsverlaufenden Muskeln, die mit der Muscularis mucosae in Verbindung stehen. Während Middeldorpf die Sehichte glatter Muskulatur Stratum submucosum, Brücke sie innere Muskelhaut nannte, gab ihr Henle (7) in seinem Handbuch der Eingeweidelehre des Mensehen 1873 den Namen Muskelschicht der Schleimhaut = Muscularis mucosae. Auch er maass diese Schicht und fand sie im Oesophagus 0,02 bis 
$0,07 \mathrm{~mm}$, im Magen $0,05-0,07 \mathrm{~mm}$, im Dünndarm $0,02 \mathrm{~mm}$. $\mathrm{Er}$ sah deutlich, dass sich aus der Muskellage der Schleimhaut die Muskellage der Zotten in Form feinster Bündel erhebe, die parallel zu einander von der Basis zur Spitze ziehen. Die Endigung der Muskelzellen erfolgt derart, dass, wie Heidenhain (8) es schildert, aus dem Bindegewebe der Muskeln feinste Fäden austreten, die mit der Spitze und Peripherie der Zotten in Verbindung stehen und, ähnlich den Sehnen, bei Contraction der Muskelbündel eine Formveränderung der Zottenoberfläche bedingen.

Somit stellt die Muscularis mucosae eine nahezu continuirliche Schicht dar, welche nach den Untersuchungen von Schaffer (9) im Oesophagus als Fortsetzung der elastischen Grenzschicht des Schlundkopfes beginnt und sich durch Magen und Darm verfolgen lässt: Sie besteht beim Menschen und den meisten Thieren aus einer inneren, ringförmig- und einer äusseren, längsverlaufenden Schicht; öfters tritt auch eine Dreitheilung der Schichten ein. So habe ich besonders schön im Magen und Darm der Katze drei deutlich von einander geschiedene Schichten erkennen können, eine innere und äussere längs- und eine mittlere ringförmig verlaufende Schicht.

Die Muscularis mucosae wird im Dünndarm durchbrochen von den Ausführungsgängen der Glandulae duodenales Brunneri. Es schien in meinen Präparaten, als ob stellenweise das ganze Convolut der Drüsen derart in die Schleimhaut eingelagert sei, dass dadurch die Muscularis eine völlige Unterbrechung erleide. Untersuchungen von Lipsky (10) haben ergeben, dass die Muscularis an diesen Stellen nur verdünnt erscheint, und dass die einzelnen Muskelfasern zwischen die Drüsenläppchen eindringen.

Eine weitere, ausgedehntere Durchbrechung erleidet die Muscularis durch die solitären Lymphknötchen und die Peyer'schen Plaques. Diese entstehen in der Tunica propria und wachsen in die Submucosa hinab, wobei sie die Muscularis durchbrechen.

Dass die Muskelschicht eine Unterbrechung zeigt an den Stellen, an denen Arterien, Venen und Lymphgefässe durchtreten, ist selbstverständlich. Bemerkenswerth ist die von Mall(11) beschriebene Thatsache, dass die Muscularis im Gebiete ihrer ringförmig verlaufenden Schicht um die Durchtrittsstelle der Venen eine Art Sphinkter bildet, der aus drei Muskelfasern besteht. Injectionsversuche haben ergeben, dass sich dieses Sphinkter contrahirte und das Lumen verengte.

E. Pflüger, Archiv für Physiologie. Bd. 98. 
Was nun das physiologische Verhalten der Muskelbündel der Schleimhaut betrifft, so hat zuerst Brücke die Erscheinung beschrieben, dass die Schleimhaut des Dünndarms, wenn man langsam mit einer geknöpften Sonde über sie streicht, einsinkt, ein granulirtes Aussehen bekommt, und erkannt, dass diese Veränderung von einer Verkürzung und Verdickung der Zotten herrührt. Auch Donders (12) beschrieb nach Brücke diese Veränderung der Zotten in Folge von mechanischer Einwirkung als bedingt durch die Contraction der Zottenmuskulatur. Veränderungen der Zottenoberfläche schildert auch Toldt (13). Fr sieht bei stärkerer Zusammenziehung mehrfach seichtere oder tiefere Einkerbungen an dem seitlichen Umfange der verbreiterten Zotte, wodurch dieselbe das schon älteren Autoren bekannte, an eine Fliegenmade erinnernde Aussehen erhält.

Durch mechanische Reizung mit spitzen Fremdkörpern treten aber, wie A. Exner schildert, am Ort der Reizung Einziehungen in der Schleimhaut auf, die von einem harten, contrahirten Muskelbündeln entsprechenden Wall begrenzt sind. Wie schon in der Einleitung gezeigt, bietet somit diese Dellenbildung, die durch die Wirkung der Muscularis mucosae hervorgerufen wird, einen wirksamen Schutz vor Verletzungen der Darmschleimhaut durch spitze Gegenstände.

Es entsteht nun die Frage, ob jene Thiere, deren Lebensweise und Ernährung es mit sich bringt, dass sie eine grosse Menge von spitzen Gegenständen verschlucken, von der Natur mit einer reichlicher entwickelten, also stärkeren Muscularis mucosae ausgestattet sind, und ob jene, die sich vorwiegend von weicher Nahrung nähren, wie viele Pflanzenfresser, in ihrem Darm eine mangelhafte Ausbildung der Muskulatur der Schleimhaut zeigen. Ferner, ob es sich bestätigt, dass jene Thiere, die, an weiche Nahrung gewöhnt, durch Zufall spitze Fremdkörper verschlucken und so häufig an Darmperforationen im Anschluss daran erkranken, wirklich eine schwächere Muscularis mucosae und somit eine ungenügende Schutzvorrichtung besitzen. Es ist nun freilich nicht zu erwarten, dass bei allen Pflanzenfressern die Muscularis mucosae dünner und schwächer entwickelt sei als bei den Raubthieren, da ja auch die ersteren mit ihrer Nahrung spitze Fremdkörper, Holzsplitter, Disteln und Stacheln aufnehmen. Es kann sich also nur darum handeln, ob sich im Allgemeinen ein Zusammenhang zwischen der Entwicklung der Muscularis mucosae und der Art der Nahrung feststellen lässt. 
Dass sich Unterschiede in der Stärke der Muscularis mucosae finden, bemerkt schon Heidenhain (14), dem es auffällt, dass gegenüber der reichlichen Ausbildung der Muskulatur und des Bindegewebsapparates in den Zotten des Hundes diese beiden Gewebe in der Darmschleimhaut des Kaninchens und Meerschweinchens sebr spärlich entwickelt sind.

Was nun die Häufigkeit des Vorkommens von Verletzungen durch spitze Fremdkörper betrifft, so zeigt sich auch hierin anscheinend ein verschiedenes Verhalten.

A. Exner bemerkt ausdrücklich die auffallende Erscheinung, dass er niemals eine der Nadeln mit ihrer Spitze in die Darmschleimhaut eingebohrt gefunden habe, obwohl er an sieben Katzen und ebenso vielen Hunde über achthundert Nadeln verfüttert hatte.

Dagegen ist die Literatur der durch Fremdkörper im Magen und Darm der Wied.erkäuer hervorgerufenen Krankheitserscheinungen eine sehr reiche. Ganz besonders ist die Haube (Netzmagen) des Rindes und der Ziege Verletzungen durch Nadeln, Nägel, spitze Knochenstücke u. s. w. ausgesetzt. Solche Nadeln gelangen oft durch das Zwerchfell in den Herzbeutel, und es ist das Vorkommen von auf diese Weise entstandenen traumatischen Pericarditiden eine bei Rind und Ziege sehr häufige, auch im Lehrbuche von Friedberger und Fröhner (15) besehriebene Erscheinung ${ }^{1}$ ). Nun sah ich, dass thatsächlich der Netzmagen des Rindes und der Ziege eine äusserst schwach entwickelte Muskulatur der Schleimhaut besitzt, die nur aus ganz vereinzelten Muskelfasern besteht. Dass auch beim Hunde Störungen durch verschluckte spitze Fremdkörper vorkommen, wird zwar in obgenanntem Werke von Friedberger und Fröhner bemerkt, gleichzeitig auch die Thatsache verzeichnet, dass solche bei der Katze viel seltener eintreten.

Ich bestimmte nun die Stärke der Muscularis mucosae im Verdauungskanal verschiedener Thiere. Zur Untersuchung kam der Magen und Darmtract von Rind, Ziege, Pferd, Schwein, Kaninchen, Meerschweinchen, Katze, Fuchs und Fischotter. Ausserdem wurde noch der Darm des Waldkauzes (Syrnium aluco) und des Hechtes (Esox lucius) untersucht und, so weit es möglich war, jedes Mal

1) Wie mir Prof. Latschenberger mitzutheilen so freundlich war, befindet sich in der Sammlung der Wiener thierärztlichen Klinik von Prof. Schindelka eine Stopfnadel, die auf diese Weise in den Herzbeutel gelangt ist. 
Oesophagus, Magen, Dünudarm, und zwar oberes, mittleres und unteres Drittel desselben, und Dickdarm einzeln geprüft. Sämmtliche untersuchten Objecte waren den frisch getödteten Thieren entnommen. Als Härtungsflüssigkeit wurde theils $M u ̈ l l ~ e r$ 'sche Flüssigkeit, theils Pikrinsäuresublimat verwendet und die Schnitte nach van Gieson gefärbt, wobei die Contrastfärbung zwischen gelber Muskulatur und rothem Bindegewebe deutlich zu Tage trat.

Was nun die Messungen selbst betrifft, so habe ich zuerst das Verhältniss zwischen Muscularis mucosae und Muscularis externa bestimmt. Unter dieser ist zusammenfassend die äussere Ring- und Längsmuskulatur verstanden. Durch solche relative Bestimmung glaubte ich mich vom jeweiligen, nach Zufälligkeiten wechselnden Contractionszustand des Darmrohres unabhängig zu machen, und am ehesten zeigen zu können, wie sich die Ausbildung der Muscularis mucosae zu der der übrigen Darmmuskulatur verhält. Dieses Verhältniss ist bei den einzelnen Thieren in den untersuchten Darmpartien nahezu constant. Die angegebenen Zahlen sind Durchschnittswerthe von einer Reihe von Einzelmessungen über die Breite der Schichten.

Sie beziehen sich bei den Wiederkäuern nur auf den Labmagen. Im Pansen des Rindes und der Ziege waren nur vereinzelte, nicht zu einer Schichte verbundene Muskelfasern zu sehen. Auch der Netz- und Blättermagen enthält spärliche Muskelfasern. (Nur in den Blättern des Blättermagens ist die Muskulatur stärker entwickelt.) Es ergeben sich für den Magen folgende in Millimetern ausgedrückte Zahlen. Dieselben sind nach der Grösse des Verbältnisses in folgender Tabelle zusammengestellt.

Musc. mucos. Musc. ext. Verhältniss

\begin{tabular}{|c|c|c|c|c|c|c|c|}
\hline Fischotter, & Fundus & . - & 0,0605 & : & 0,231 & $=$ & $1: 3,8$ \\
\hline Fischotter, & Pylorus & . & 0,044 & : & 0,297 & $=$ & $1: 7$ \\
\hline Fuchs . & . & . & 0,044 & $:$ & 0,396 & $=$ & $1: 9$ \\
\hline Schwein & & & 0,066 & $:$ & 0,704 & $=$ & $1: 10,6$ \\
\hline Katze & • & . & 0,077 & $:$ & 0,902 & $=$ & $1: 11,7$ \\
\hline Kaninchen & & & 0,0176 & $:$ & 0,396 & $=$ & $1: 22,5$ \\
\hline Pferd. . & & . & 0,088 & : & 2,2 & $=$ & $1: 25$ \\
\hline Rind & & & 0,0264 & : & 0,77 & $=$ & $1: 28$ \\
\hline Ziege & . . & & 0,022 & : & 0,99 & $=$ & $1: 45$ \\
\hline
\end{tabular}

Beim Meerschweinchen liess sich ein Verhältniss nicht aufstellen, da die Muscularis mucosae bloss aus einzelnen Fasern besteht. 
Es zeigt sich also, dass ein ganz bedeutender Unterschied in dem Verhalten der Muscularis mucosae zu der Muscularis externa bei den vier erstangeführten Thieren im Vergleich zu den sechs letztgenannten besteht. Während die Muskularis mucosae bei diesen nur den 22.-45. Theil der äusseren Muskulatur misst, ist sie bei Fischotter, Fuchs, Schwein und Katze bedeutend stärker und bildet den 4.-11. Theil der Muscularis externa.

Ich habe ferner die absoluten Werthe der Muscularis mucosae (an den gehärteten Präparaten) bestimmt und mit einander verglichen, wobei ich auch die Angaben Mall's (16) über die Stärke der Muscularis mucosae im Magen des Hundes und diejenigen Brümmer's (17) über ihre Stärke bei der Feldmaus und Wasserratte heranzog. Diese Zahlen sind nach der absoluten Dicke der Muscularis mucosae geordnet.

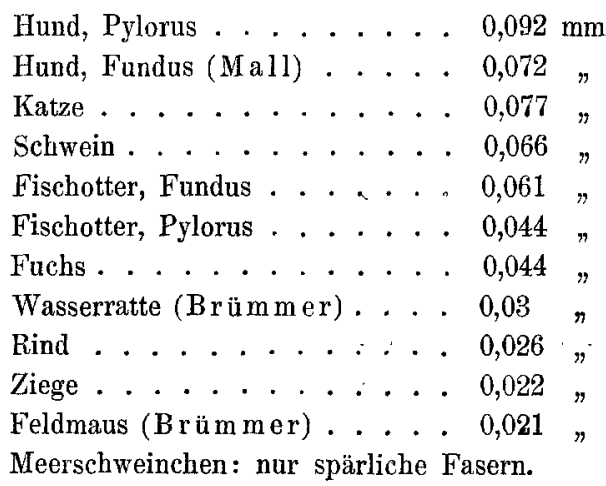

Auch hierin zeigt sich wieder ein Ueberwiegen der Muscularis musosae - wenn auch nicht in so auffallender Weise - bei Fuchs, Fischotter, Schwein, Katze und Hund gegenüber der der anderen untersuchten Thiere.

Man sieht, dass auch in dieser Reihe, bei der die absolute Grösse der Thiere ausser Betracht bleibt, diejenigen oben stehen, deren Magen stärker gefährdet ist, diejenigen unten, bei denen dieses in geringerem Maasse der Fall ist. Die letztgenannten nähren sich zuineist von weicher Pflanzenkost, die erstgenannten, vorwiegend Fleischfresser, von einer Kost, die zahlreiche spitze Gegenstände, Gräten, Knochenstücke $u$. dgl., einschliesst. Somit scheint thatsächlich zwischen der Ausbildung der Muscularis mucosae und der Art der Nahrung der Thiere ein derartiger Zusammenhang zu bestehen, dass diejenigen Thiere, die mit ihrer Nahrung spitze Gegenstände zu sich 
nehmen, eine stärkere Muscularis mucosae und mit ihr eine vollkommenere Schutzvorrichtung vor Verletzungen besitzen.

Wie sich die Muscularis mucosae in den einzelnen Altersstufen des Menschen verhält, darüber hat Baginsky (18) Untersuchungen angestellt. Wiewobl sich aus seinen Zahlen nicht gerade deutlich ein Wachsthum der Muscularis mucosae erkennen lässt, behauptet doch Baginsky, dass die Stärke dieser Schicht innerhalb der von ihm untersuchten Zeitabschnitte, d. i. vom viermonatigen Embryo bis zum dreijährigen Kinde, zunehme. Diese Thatsache kann mit dem allgemeinen Wachsthum des Individuums erklärt werden; vielleicht ist aber auch in der stärkeren Entwicklung dieser Schichte eine Anpassung der Muscularis mucosae an die resistentere Nahrung des Kindes gegenüber der Milehernährung des Säuglings zu sehen.

Meine Untersuchungen des Darmes bezogen sich auf das obere, mittlere und untere Drittel desselben und auf den Dickdarm. Die Messungsresultate, in Millimetern ausgedrückt, sowie das Verhältnis der beiden Muskelschichten waren, nach der Grösse dieses Verbältnisses geordnet, folgende:

\section{Dünndarm (erstes Drittel).}

Musc. mucos. Musc. ext. Verhältniss

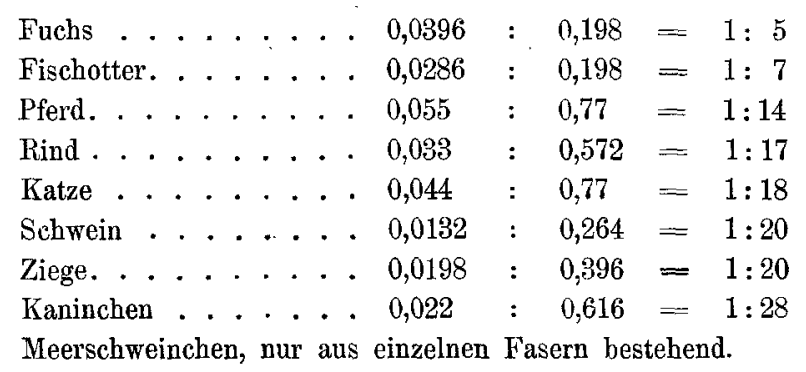

Auch im oberen Theile des Dünndarms zeigt sich somit ein ähnliches Verhalten zwischen Muscularis mucosae und externa wie im Magen. Es ist die Muskelschicht der Schleimhaut bei Fuchs und Fischotter viel stärker entwickelt als bei den übrigen Thieren.

Die absoluten Zahlen für die Dicke der Muscularis mucosae ergeben folgende Werthe:

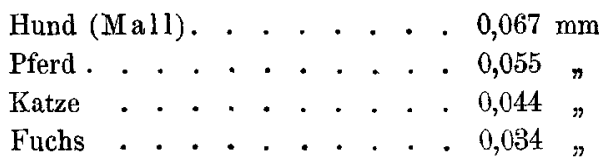


Das anatomische Verhalten der Muscularis mucosae etc.

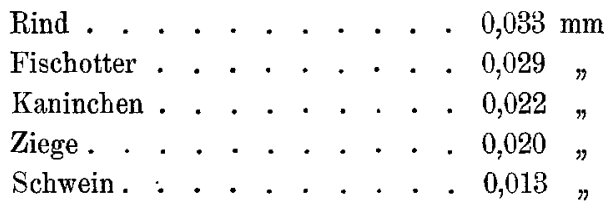

Meerschweinchen, nur aus einzelnen Fasern bestehend.

Wenn auch nicht so deutlich, so zeigt sich auch in diesen Werthen ein Ueberwiegen der Muscularis mucosae bei Hund, Katze, Fuchs and Fischotter. Allerdings steht das Rind auch hier in der Reihe ziemlich hoch.

Dünndarm (mittl. Drittel).

Musc. mucos. Musc. ext. Verhältniss

Fuchs. . . . . . . fehlt

Schwein . . . . . . 0,0242 : $0,264=1: 11$

Kaninchen . . . . 0,0066 : $0,088=1: 18$

Fischotter . . . $0,0286: 0,418=1: 15$

Rind . . . . . . $0,044: 0,66=1: 15$

Katze . . . . . . 0,033 : $0,66=1: 20$

Pferd . . . . . . $0,044: 1,012=1: 23$

Ziege . . . . . . $0,028: 0,902=1: 32$

Meerschweinchen, keine deutliche Schicht.

Die absoluten Werthe, in eine Reihe gestellt, ergaben:

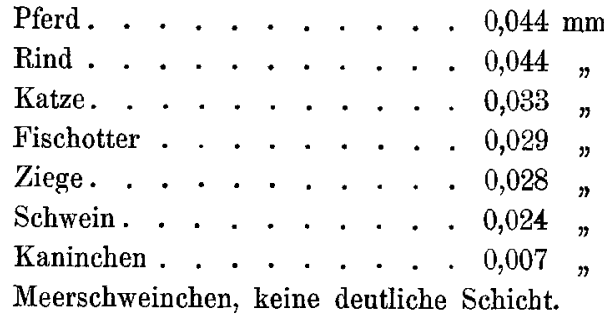

Dünndarm (unteres Drittel).

Musc. mucos. Musc. ext. Verhältniss

Fuchs . . . . .. . fehlt

Kaninchen . . . $0,0044: 0083=1: 8$

Fischotter . . . . $0,022: 0,275=1: 13$

Pferd . . . . . . $0,022: 0,297=1: 14$

Ziege . . . . . $0,011: 0,176=1: 16$

Rind . . . . . . $0,022: 0,572=1: 26$

Schwein . . . . $0,022: 0,605=1: 28$

Katz: . . . . . 0,083 : $0,964=1: 29$

Meerschweinchen, keine deutliche Schicht. 
Die absoluten Werthe ergaben:

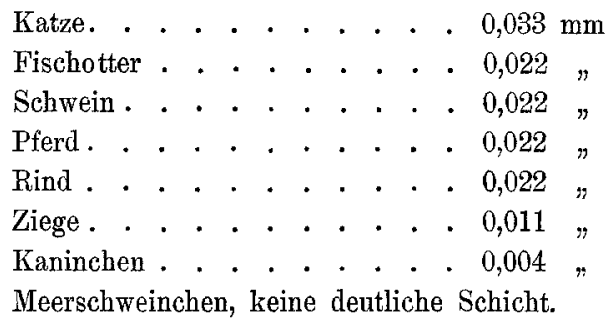

Im zweiten und dritten Drittel des Dünndarms lässt sich somit kein auffallender Unterschied erkennen. Immerhin nimmt die Fischotter durch die Entwicklung ihrer Schleimhautmuskulatur bei Bestimmung sowohl der absoluten wie relativen Werthe eine der ersten, Ziege und Meerschweinchen eine der letzten Stellen ein.

Was nun die relativen Werthe der Muscularis mucosae im Dickdarm betrifft, so ergeben sich folgende Werthe:

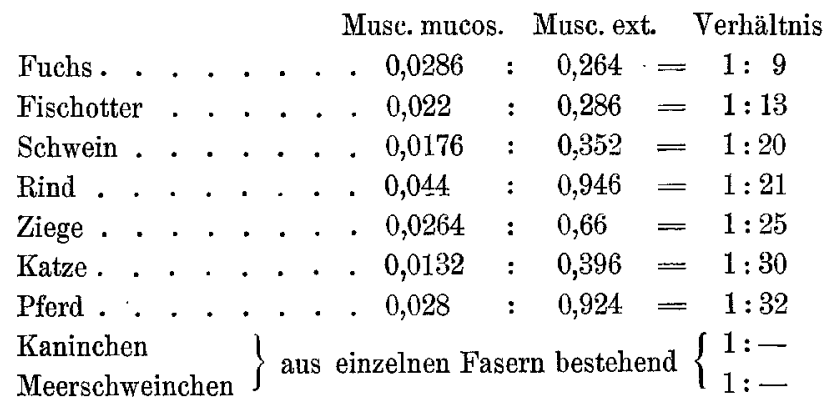

Wieder erscheint bei Fuchs und Fischotter die Muscularis mucosae stärker entwickelt und beträgt bei diesen den 9.--13. Theil der äusseren Muskelschicht.

Die absoluten Werthe im Dickdarm, ihrer Grösse nach eingereiht, sind :

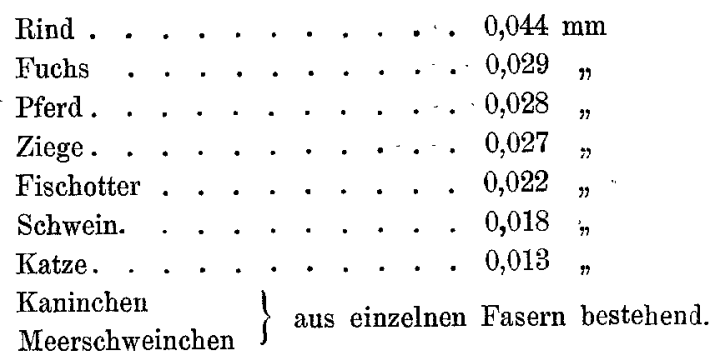


Die absoluten Zahlen lassen sonach im Dickdarm keine Anordnung in dem Sinne erkennen, dass bei den Raubthieren die Werthe grösser als bei den anderen untersuchten Thieren wären. Sie sind bei den meisten Thieren nahezu gleich gross.

Die Resultate sind daher folgende:

Die Muscularis mucosae ist im Magen und im oberen Antheile des Dünndarms deutlich stärker entwickeit bei den Thieren, deren Verdauungstract einer Gefährdung durch spitze Fremdkörper ausgesetzt sind, als bei denen, die sich von weicher Kost nähren. Dieser Unterschied tritt besonders deutlich beim Vergleich der Fischotter, die mit ihrer Fischnahrung zahlreiche Gräten verschluckt, mit der Ziege, die hauptsächlich weiche Pflanzenkost zu sich nimmt, hervor. Im unteren Abschnitte des Dünndarms und im Dickdarm ist diese Verstärkung der Muskelschicht der Schleimhaut - eine Art Arbeitshypertrophie -- nicht nachweisbar. Es zeigt sich ferner im Allgemeinen eine Abnahme der Dicke der Muscularis mucosae vom Magen zum Dickdarm.

Die Thatsache, dass die unteren Darmpartien eine viel schwächere Muscularis mucosae besitzen als die oberen, und dass hier die Abhängigkeit der Stärke von der Art der Nahrung weniger auffallend ist, ist damit zu erklären, dass Dickdarm und Ileum einer Läsion durch spitze Gegenstände weniger ausgesetzt sind als die oberen Iarmabschnitte, da solche Fremdkörper. wie Gräten, die im Magen und Dünndarm leicht Verletzungen hervorrufen könmen, auf ihrem Wege durch den Verdauungseanal theils durch dessen Secrete erweicht und auf diese Weise abgestumpft, theils in feste Skybala eingeschlossen und so unschädlich gemacht werden.

Ich füge noch die Befunde am Oesophagus einiger Thiere hinzu, obwohl die Muscularis mucosae hier weniger als Schutzvorrichtung dienen wird, da ja die Speiseröhrenschleimhaut durch ihr mächtiges Plattenepithel einigermassen vor Verletzungen geschützt ist.

$$
\text { Oesophagus: }
$$

Dicke des Muscularis mucosae bei

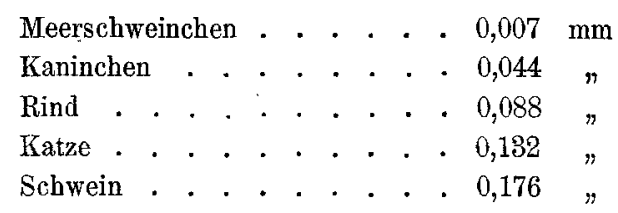


Während demnach der Oesophagus wesentlich durch sein dickes Epithel vor Verletzungen geschützt zu. sein scheint, ist dieser Schutz im Magen und im oberen Theil des Dünndarmes durch die Thätigkeit der Muscularis mucosae ersetzt. Es kommt dann noch ein weiterer, bis jetzt in dieser Beziehung nicht beachteter Factor hinzu. Es ist dies die sogen. Zeisel'sche Membran. Während Brücke schon in seiner ersten Arbeit über die Muscularis mucosae erklärt hatte, dass die für die Schleimhant der Zotten bestimmten Muskelbündel von der Muscularis mucosae geliefert werden, eine Ansicht, der sich auch Toldt(19), Kölliker (20) in seinem von Ebner bearbeiteten Handbuch und viele Andere anschlossen - auch Stöhr (21) schreibt: Die Muscularis mucosae besteht aus glatten Muskelfasern, von denen sich im Magen einzelne Züge abzweigen, um in senkrechter Richtung zwischen die Drüsenschläuche emporzusteigen; im Darm reichen senkrecht von der Muscularis mucosae aufsteigende Fasern bis nahe zur Spitze der Zotte -, haben sich andere Forscher, Mall (22) und Roszner (23) gegen einen Zusammenhang dieser beiden Muskelsysteme ausgesprochen, und zwar aus folgendem Grunde: Die Schleimhaut des Magens und Darmes besteht nach den Angaben von Mall aus dem Epithel, einer dünnen, die Grenzschicht bildenden Membran, der eigentlichen Schleimhaut, dem Stratum granulosum, dem Stratum fibrosum und der Muscularis mucosae. Zwischen eigentlicher Schleimhaut und Museularis mucosae ist somit bei gewissen Thieren eine Schicht eingelagert, die zuerst von Langer und Molin genannt und von M. Zeisel näher beschrieben wurde. Zeisel (24) fand im Magen, sonst aber in keinem Darmabschnitt, der Katze eine Schicht, die er ebenso wie Glinsky (25) als glasartig bezeichnete, welche annähernd dieselben chemischen Reactionen gibt wie Bindegewebe, aus in steilen Wellen gelegten Faserzügen besteht und kleine, kernarme Gebilde erkennen lässt. Diese von Mall (26) als Stratum fibrosum, von Oppel (27) als Stratum compactum bezeichnete Schicht ist es, die nach Mall eine Verbindung zwischen den Muskelelementen der Zotten und denen der Muscularis mucosae ausschliesst und ihr ein „unübersteigbares Hinderniss" entgegensetzt. Mall behauptet sonach, dass die Muskeln der Zotte - allerdings oft schon tief - in der Kryptenschicht beginnen und in den Zotten endigen.

Im Magen des Falkens und der Katze wurde diese Schicht beschrieben. Auch im Magen des Hundes hat Mall eine solche ge- 
sehen. "This rudimentary stratum fibrosum of the dog's stomach may possibly be homologized with a similar membrane described by Zeisel in the cat's stomach." Sie ist ferner in grösserer oder geringerer Ausbildung im Darm von Hecht, Huchen, Forelle, Schleie, Manis javanica, Dasyurus hallucatus, Hund und Fuchs beschrieben worden. Ich habe in meinen Schnitten diese Schicht ebenfalls als eine scharf abgegrenzte, wellig verlaufende, kernarme, durch Säurefuchsin sich intensiv röthlich färbende Schicht gesehen, die an Dicke ca. $0,022 \mathrm{~mm}$ beträgt. Im Magen der Katze, im Darm des Fuchses, des Hechtes und dem des Waldkauzes war sie deutlich sichtbar, dagegen nicht in dem der Fischotter oder der übrigen untersuchten Thiere.

Oppel deutet die Schichte derart, dass in diesem Stratum compactum das gesammte Stützgewebe des Darmes seine stärkere Entwicklung, gewissermaassen seine Grundlage und seinen Hauptstützpunkt, fnde, und ihre Bedeutung mag darin bestehen, dass sie die Wirkung der Muscularis mucosae zu ergänzen oder zu ersetzen vermag. Die Entstehung solcher Membranen scheine einen $\mathrm{Zu}-$ sammenhang mit der Muscularis mucosae zu haben.

Nun scheint es doch beachtenswerth und auffallend, dass gerade bei jenen Thieren, die, wie Fuchs, Katze und Hecht, nach meinen Messungen eine stärkere Muscularis besitzen und deren Schleimhaut des Darmtractes der Gefährdung durch spitze Gegenstände besonders ausgesetzt ist, auch das Stützgerüst zu einer compacten, sehnenartigen, resistenteren Schicht umgewandelt ist. Es scheint somit, als würde der Darmtract nicht nur durch die Muscularis mucosae vor Verletzungen geschützt sein, sondern, dass im Falle eine solche durch Eindringen eines spitzen Körpers doch eingetreten wäre, das Durchdringen desselben bis an das Peritoneum oder gar in die Bauchhöhle durch die aponeurotische Sehicht hintangehalten würde.

Zum Schlusse sei es mir gestattet, dem Vorstand des physiologischen Institutes, Herrn Prof. Sigm. Exner, für die Wahl des zu bearbeitenden Themas, und dem Assistenten Herrn Dr. Const. E c o n o mo für seine unermüdliche Unterstützung bei der Ausführung der Untersuchung den besten Dank zu sagen. 
402 Bianca Bienenfeld: Das anat. Verhalten der Muscularis mucosae.

\section{L i t e r a t n r.}

1) Dr. A. Exner, Wie schützt sich der Verdauungstract. Pflüger's Archiv f. Physiol. Bd. 89. 1902.

2) A. Th. Middeldorpf, Disquisitio de glandulis Brunnianis Vratislaviae. 1846.

3) Lacauchie, Compt. rend. de l'Acad. de Paris t. 16. 1843.

4) Gruby u. Delafond, Compt. rend. t. 16.

5) E. Brücke, Das Muskelsystem der Schleimhaut, des Magens und Darmcanals. Sitzungsber. der Wiener Akad. d. Wissensch. Bd. 6. 1851.

6) Kölliker, Zeitschr. für wissenschaftl. Zoologie Bd. 3. 1851.

7) Henle, Handbuch der Eingeweidelehre der Menschen Bd. 2. 1873.

8) Heidenhain, Beiträge zur Histologie and Physiologie der Dünndarmschleimhaut. Pflüger's Archiv f. Physiol. Bd. 43. 1888.

9) Schaffer, citirt bei Kölliker, Handbuch der Gewebelehre. Sitzungsber. d. Akad. d. Wissensch. Bd. 100. 1902.

10) Lipsky, Beiträge zur Kenntniss des feinen Baues des Darmcanals. Sitzungsber. d. Wiener Akad. d. Wissenseh. Bd. 55. 1867.

11) Mall, Die Blut- und Lymphwege im Dünndarm des Hundes. Abh. d. math. phys. Classe d. kgl. sächs. Ges. d. Wissensch. Bd. 16. 1888.

12) Donders, Physiologie des Menschen Bd. 1. 1856.

13) C. Toldt, Lehrbuch der Gewebelehre. 1888.

14) Heidenhain, siehe (8).

15) Friedberger und Fröhner, Ueber spec. Pathol. und Therapie der Hausthiere. 1886.

16) Mall, Vessels and Walls of the dog's stomach. John Hopkins Hosp. Rep. Bd. 5. (1). 1892.

17) Brümmer, Anatom. u. hist. Untersuch. über d. zusammeng. Magen versch. Säugeth. Deutsche Zeitschr. f. Thiermedicin 1876.

18) Baginsky, Unters. üb. d. Darmcanal d. menschl. Kindes. Virchow's Archiv Bd. 89. 1882.

19) C. Toldt, siehe (13).

20) Kölliker, Handbuch der Gewebelehre, bearbeitet durch von Ebner. 1902.

21) Ph. Stöhr, Lehrbuch der Histologie. 1903.

22) Mall, siehe (11).

23) A. Roszner, Beiträge zur Histologie des Dünndarmes. Ungar. Archiv f. Medicin Bd. 3. 1895.

24) M. Zeisel, Ueber eine eigenthümliche Schicht im Magen der Katze. Sitzungsber. d. Akad. d. Wissensch. Wien. 1875 Abth. 8.

25) Glinsky, Zur Kenntniss des Baues d. Darmschleimhaut der Wirbelthiere. Centralbl. f. d. med. Wissensch. 1883 Nr. 13.

26) Mall, siehe (16).

27) Oppel, Lehrbuch d. vergl. mikr. Anatomie. 1902. 